\title{
Research and realization of 3D digitization of static fabric based on Gray code
}

\author{
Jian $\mathrm{Li}^{1, \mathrm{a}}$, Xirui $\mathrm{Du}^{1, \mathrm{~b}}$, Xiaojiao Tao ${ }^{1, \mathrm{c}}$ and Chengyan Zhang ${ }^{1}$ \\ ${ }^{1}$ College of Electrical and Information Engineering, Shaanxi University of Science \& Technology, \\ Xi'an 710021, China \\ alijianjsj@sust.edu.cn, \\ bduxirui@163.com \\ ctaoxiaojiao@sust.edu.cn
}

Keywords: Binocular stereo vision, Camera calibration, Gray code, Stereo Matching, 3D reconstruction.

Abstract. Combined with the Gray-encoding structured light and binocular stereo vision, the 3D digital reconstruction of the static fabric is realized in this paper. Zhang Zhengyou camera calibration method is adopted to achieve camera's stereo calibration. After choosing effective area and binarization, the images are decoded by the Gray code, thus an area matching strategy is proposed to achieve matching uniqueness, and the 3D digitalization of fabric is achieved based on binocular stereo vision. The reconstruction result demonstrates accuracy and reliability of proposed method.

\section{Introduction}

3D measurement system with structured light has advantages of rapid, accurate and low cost, and is widely used in reverse engineering, the protection of cultural relics and other fields[1]. Classical triangulation method is used to calculate the $3 \mathrm{D}$ coordinates of the sampling point of object surface. The method shortens the measure time and measurement efficiency is promoted.

Gray code is widely used, which possesses preferable antijamming ability, and coding simplicity. Bergmann et al.[2] combined advantages of Gray code and phase-shifting code, proposed a integration of Gray code and phase-shifting coding algorithm. Lin et al.[3] proposed a novel method based on plaster model of the human eyes. In [4], Hou presented a coding method based on 7-bit Gray code and 16-times Phase Shifting to realize the reconstruction of 3D point clouds. But the method needs more images, more digit code, and is prone to error.

This paper combined the Gray-encoding structured light with binocular stereo vision to achieve the $3 \mathrm{D}$ reconstruction of the static fabric. And Zhang Zhengyou camera calibration method[5] is adopted to realize the camera's stereo calibration. The proposed method test on sheets, shirts, pants and skirts, the results show that the proposed method presents high precision.

\section{Coded image acquisition}

The background is black in order to choose effective area effectively and improve the computing speed. Tests would mainly be carried out on sheets, shirts, pants and skirts.

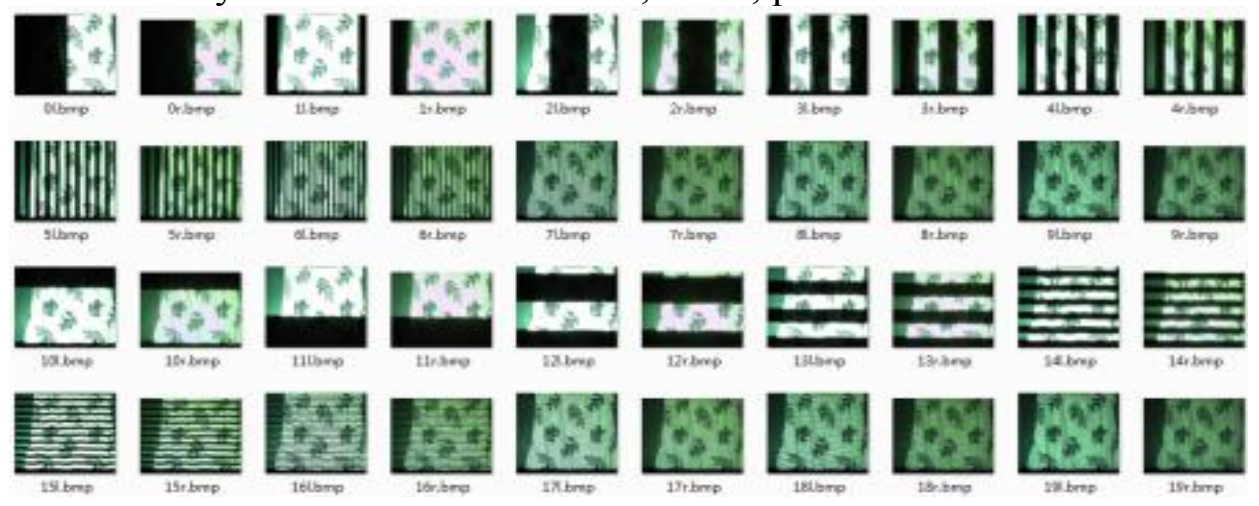

Fig.2 The sheet images acquired by cameras 
20 horizontal and vertical Gray code images and complement code images are projected onto the surface of measured object, then acquiring the code image pairs by double cameras and stored in the computer. As shown in Fig.2, odd column image pairs of sheets are captured by left camera, even column are captured by right camera.

\section{Coded image preprocessing}

Effective area selection. The biggest gray value difference between all white images and all black images captured by left camera is T. In this paper, the threshold is 0.25 times of maximum value. The process of selecting effective area is shown in Fig.3, the first line are black images, the second line are white images and the third are effective areas. It can be concluded from these results that in black background blind area can well be removed. Stereo matching and 3D reconstruction only in effective areas can improve the computation of 3D digitization.

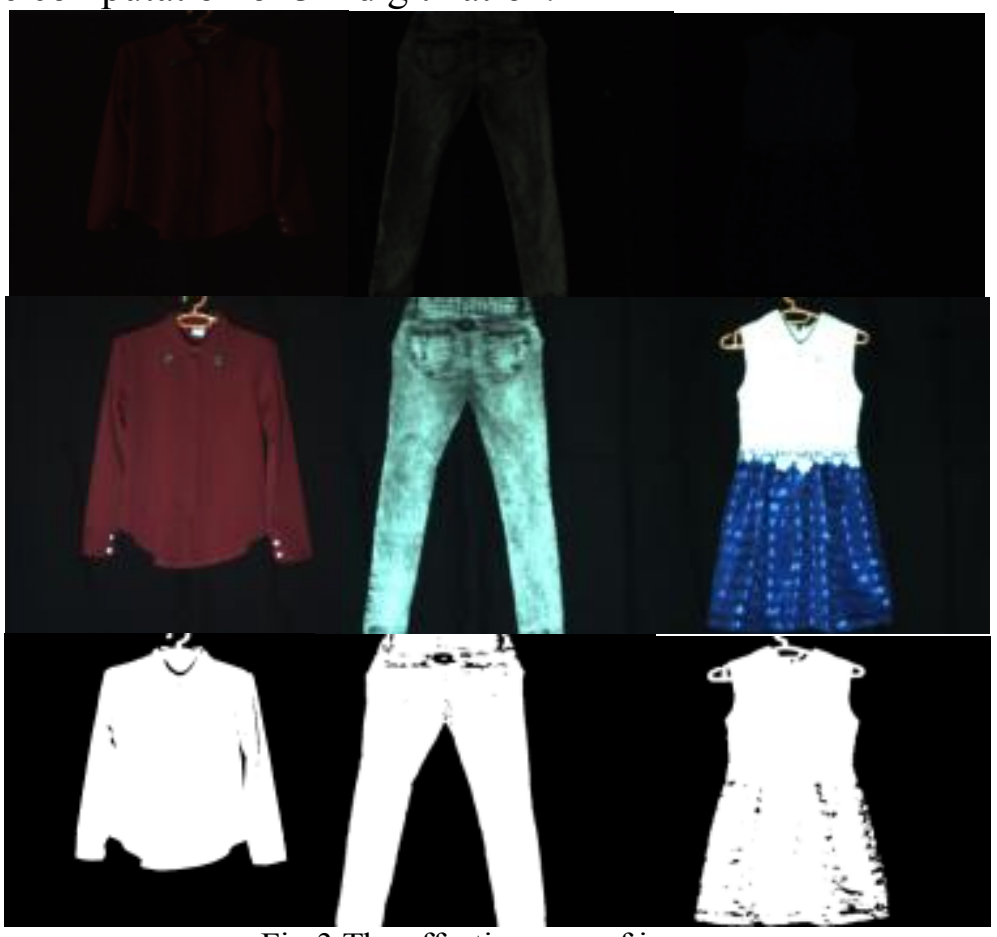

Fig.3 The effective area of image

Encoded image binarization. In this paper, a simplified double threshold segmentation method[6] is applied. The specific process is: given a point $M(x, y)$, comparing the grey value of $M(x, y)$ in the Gray code image and Gray's complement code image. Assume that $M^{+}(x, y)$ is the gray value in the Gray code image and $M^{-}(x, y)$ is the gray value in the Gray's complement code image. If $M^{+}(x, y)>M^{-}(x, y), M(x, y)=1$, otherwise $M(x, y)=0$. The experimental results of adaptive threshold segmentation shirt is shown in the Fig.4, and Fig.5 shows the effect of proposed method of segmentation. It can be concluded from the result that the proposed method can segment the texture information of measured object well.
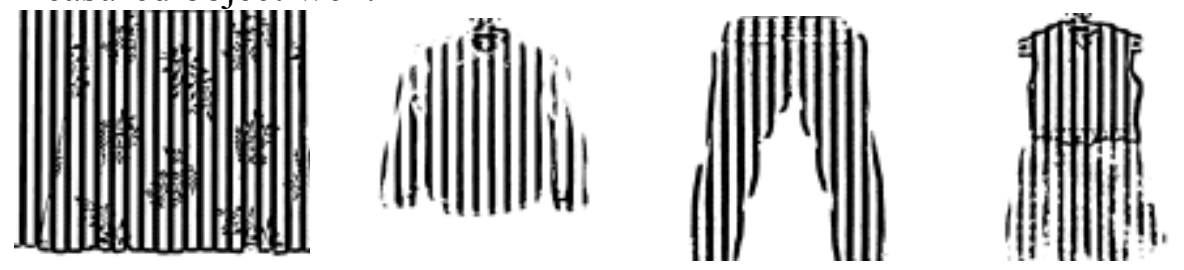

Fig.4 Adaptive threshold segmentation method 


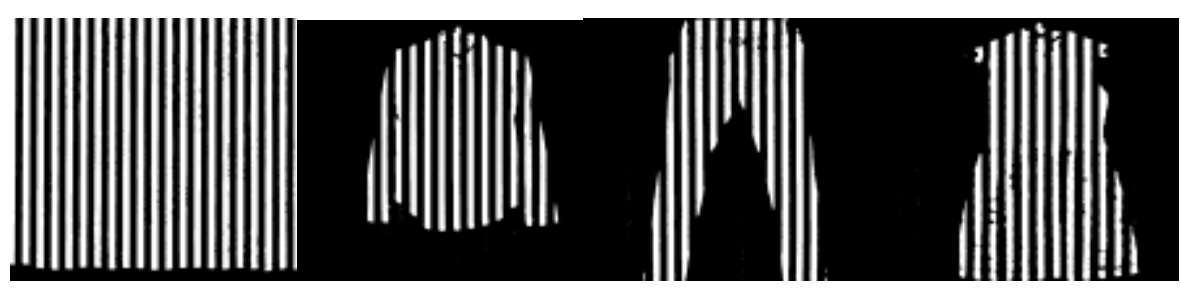

Fig.5 Proposed method segmentation

Stripe encoding. If the pixels have the same code and the variance of them is less than threshold, these points are considered in the same area. Similarly, the variance and mean value of the pixels are calculated in the right camera, and judging whether these points in a same area according to the threshold. The mean value of the region is regarded as right image matching points in left image, thus realizing the uniqueness of the matching. Compared with the feature matching method, the proposed method can almost find all the points on the measured object, have high matching accuracy, and is easy to achieve.

The acquisition of 3D point cloud. After got the matching points from the two images, we combine it with the camera calibration information. Using the classic Triangle Measuring Method, we can get the depth information by solving a equation set. Assuming that $\boldsymbol{P}$ is a point on the object and $\boldsymbol{p}_{l}, \boldsymbol{p}_{r}$ are the projection points on the image plane in two cameras. $\boldsymbol{M}_{l}, \boldsymbol{M}_{r}$ are the projection matrixs of the left camera and the right camera respectively. We can get the following equation:

$$
\lambda_{l}\left[\begin{array}{c}
u^{l} \\
v^{l} \\
1
\end{array}\right]=\left[\begin{array}{llll}
p^{l} & p_{12}^{l} & p_{13}^{l} & p^{l}{ }_{14} \\
p^{l} & p^{l}{ }_{22} & p^{l}{ }_{23} & p^{l}{ }_{24} \\
p_{31}^{l} & p^{l}{ }_{32} & p^{l}{ }_{33} & p^{l}{ }_{34}
\end{array}\right]\left[\begin{array}{c}
X \\
Y \\
Z \\
1
\end{array}\right] .
$$

Expanding equation 1,we get:

$$
\begin{aligned}
& \lambda_{l} u^{l}=p_{11}^{l} X+p_{12}^{l} Y+p_{13}^{l} X+p_{14}^{l} \\
& \lambda_{l} v^{l}=p_{21}^{l} X+p_{22}^{l} Y+p_{23}^{l} X+p_{24}^{l} . \\
& \lambda_{l}=p_{31}^{l} X+p_{32}^{l} Y+p_{33}^{l} X+p_{34}^{l}
\end{aligned}
$$

Dividing the first two equation by the third equation to eliminate $\lambda_{1}$

$$
\begin{aligned}
& \left(p_{11}^{l}-u^{l} p_{31}^{l}\right) X+\left(p_{12}^{l}-u^{l} p_{32}^{l}\right) Y+\left(p_{13}^{l}-u^{l} p_{33}^{l}\right) Z=u^{l} p_{34}^{l}-p_{14}^{l} . \\
& \left(p_{21}^{l}-v^{l} p_{31}^{l}\right) X+\left(p_{22}^{l}-v^{l} p_{32}^{l}\right) Y+\left(p_{23}^{l}-v^{l} p_{33}^{l}\right) Z=v^{l} p_{34}^{l}-p_{24}^{l} .
\end{aligned}
$$

We can also get equation 4 when there are cameras:

$$
\begin{aligned}
& \left(p_{11}^{r}-u^{r} p_{31}^{r}\right) X+\left(p_{12}^{r}-u^{r} p_{32}^{r}\right) Y+\left(p_{13}^{l}-u^{l} p_{33}^{l}\right) Z=u^{l} p_{34}^{l}-p_{14}^{l} . \\
& \left(p_{21}^{r}-v^{r} p_{31}^{r}\right) X+\left(p_{22}^{r}-v^{r} p_{32}^{r}\right) Y+\left(p_{23}^{r}-v^{r} p_{33}^{r}\right) Z=v^{r} p_{34}^{r}-p_{24}^{r} .
\end{aligned}
$$

Combine equation 3 and equation 4 we get equation 5:

$$
\left[\begin{array}{ccc}
p_{11}^{l}-u^{l} p_{31}^{l} & p_{12}^{l}-u^{l} p_{32}^{l} & p_{13}^{l}-u^{l} p_{33}^{l} \\
p_{21}^{l}-v^{l} p_{31}^{l} & p_{22}^{l}-v^{l} p_{32}^{l} & p_{23}^{l}-v^{l} p_{33}^{l} \\
p_{11}^{r}-u^{r} p_{31}^{r} & p_{12}^{r}-u^{r} p_{32}^{r} & p_{13}^{r}-u^{r} p_{33}^{r} \\
p_{21}^{r}-v^{r} p_{31}^{r} & p_{22}^{r}-v^{r} p_{32}^{r} & p_{23}^{r}-v^{r} p_{33}^{r}
\end{array}\right]\left[\begin{array}{c}
X \\
Y \\
Z
\end{array}\right]=\left[\begin{array}{c}
u^{l} p_{34}^{l}-p_{14}^{l} \\
v^{l} p_{34}^{l}-p_{24}^{l} \\
u^{r} p_{34}^{r}-p_{14}^{r} \\
v^{r} p_{34}^{r}-p_{24}^{r}
\end{array}\right] .
$$

By solving the equation 5, we can get the $3 \mathrm{D}$ coordinates of the object. In this paper, we solve this equation using Singular Value Decomposition method.

\section{The experiment results}

To test and verify the proposed 3D reconstruction method, we build a binocular measurement system based on Gray code. The Gray code will be projected on the measured object by the projector. We use a $\mathrm{PC}$ to control the cameras and store the photo, and choose $\mathrm{C}++$ to write the software, using a library called OpenCV to help processing the image data. And Meshlab shows the point cloud at last. We 
reconstructed shirts, pants, skirts and other flexible fabric in proposed method. And Fig.6 shows the result in Meshlab. The first column are original images. The second column are reconstructed objects. The third are point clouds. The fourth are point clouds in different viewpoints. The reconstruction result demonstrates accuracy and reliability of the proposed method.
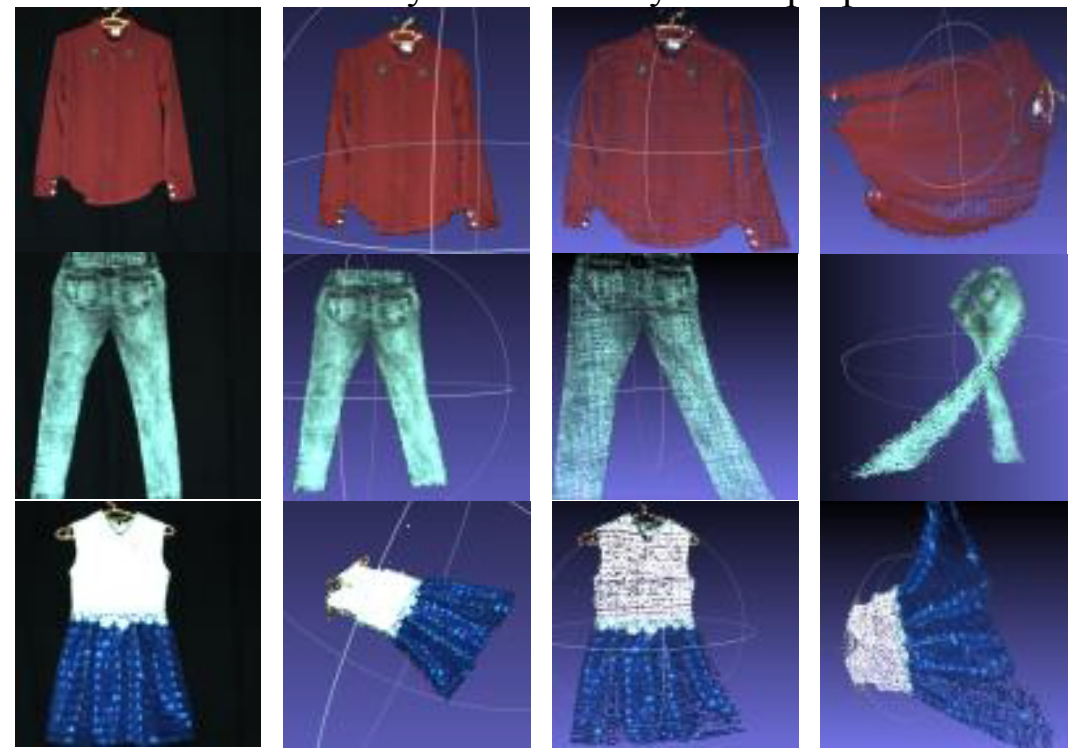

Fig. 6 The result of construction

\section{Conclusions}

The paper studies the whole process of 3D digitization of static fabric based on Gray Code. Firstly introduce the Gray code and the generation process of Gray code graph. After preprosessing decoding by the Gray code, an area matching method is utilized to achieve uniqueness of matching. The process of acquiring 3D point cloud based on Gray Code is described. The experiments have been conducted on reconstructing shirts, pants, skirts and other flexible fabric in proposed method, and show the accurate, efficient and reliability.

\section{Acknowledgements}

This work was financially supported by the Shaanxi Province Training Programs of Innovation and Enterpreneurship for undergraduates (201410708030).

\section{References}

[1] F Zollner, V Matusevich, R Kowarschik. 3D Measurement by Stereophotogrammetry[J]. Proceedings of SPIE, 2003, 5144:311-314

[2] D Bergmann. New approach for automatic surface reconstruction with coded light[J]. Proc SPIE, 1995,2572:2-9.

[3] H Lin, Z F Ma, C H Yao. 3D measurement technology base on binocular vision using a combination of Gray code and phas-shift structured light [J].Acta Electronica Sinica. 2013,41(1):24-28.

[4] F Z Hou. Research on Gray Code Composite Pattern Structured Light Illumination[D]. Xidian University, 2011.

[5] Z Y Zhang. A Flexible New Technique for Camera Calibration [J]. IEEE Transactions on Transactions on Pattern Analysis and Machine Intelligence. Vol. 22, 2000.

[6] Daniel Scharstein, Richard Szeliski. Hight-accuracy stereo depth maps using structured Light[A]. IEEE Computer Conference on Computer Vision and Pattern Recognition(CVPR). Rosten, VA, USA:IEEE. Press,2003,195-202. 